\title{
Semi-empirical Model of Global Exposure using Stochastic Geometry
}

\author{
Quentin Gontier \\ Wireless Communications Group \\ Université Libre de Bruxelles \\ Brussels, Belgium \\ quentin.gontier@ulb.be \\ François Horlin \\ Wireless Communications Group \\ Université Libre de Bruxelles \\ Brussels, Belgium \\ Francois.Horlin@ulb.be \\ Philippe De Doncker \\ Wireless Communications Group \\ Université Libre de Bruxelles \\ Brussels, Belgium \\ pdedonck@ulb.ac.be
}

\author{
Luca Petrillo \\ Département Technologies et Rayonnement \\ Brussels Environnement \\ Brussels, Belgium \\ lpetrillo@environnement.brussels
}

\author{
Joe Wiart \\ LTI, Telecom Paris, Chaire C2M \\ Institut Polytechnique de Paris \\ Palaiseau, France \\ joe.wiart@telecom-paris.fr
}

\author{
François Rottenberg \\ Wireless Communications Group \\ Université Libre de Bruxelles \\ Brussels, Belgium \\ francois.rottenberg@ulb.ac.be \\ Claude Oestges \\ ICTEAM Institute \\ Université Catholique de Louvain \\ Louvain-la-Neuve, Belgium \\ claude.oestges@uclouvain.be
}

\begin{abstract}
Exposure to electromagnetic fields due to cellular networks in an urban environment is studied using stochastic geometry. We describe a simple but functional and realistic semi-empirical model based on the modeling of base station patterns as Poisson Point Processes and applied to two Brussels municipalities. Using this model, a study of the impact of base station densification is performed according to two theoretical scenarios. From simple assumptions, it is concluded that global exposure is expected to remain approximately constant in the case of intelligent network evolution involving the densification of macro cells and the addition of a network of low-power small cells.
\end{abstract}

Index Terms-EMF exposure, stochastic geometry, cellular networks, small cells

\section{INTRODUCTION}

Stochastic geometry (SG) has been used for several years in telecommunications. It provides information on network performance that other, more classical, deterministic methods are struggling to obtain. SG enables the modeling of base station (BS) distributions in wireless networks as point processes in order to characterize distance distributions [1], signal-tointerference-plus-noise-ratio and its related performance metrics [2], [3] or localization performance [4]. The statistical nature of this approach makes it possible, for example, to elaborate stochastic models for heterogeneous cellular networks [5] or to study probabilities of coverage when one model parameter changes, such as the height of the antennas [6]. In recent years, stochastic geometry has also been used to model

This work was supported in part by the FNRS under MUSE-WINET EOS grant and Innoviris under STOEMP-EMF grant. unmanned aerial vehicles [7], [8]. In [9] and [10] SG has been recently applied to the study of exposure to electromagnetic fields. They are interested in certain frequency bands and not in the entire cellular spectrum as in this paper.

Exposure due to cellular networks is difficult to assess deterministically within a reasonable time frame, and is subject to many uncertainties (due to the number of BSs in operation, the geometry of the environment, the presence of people and vehicles causing shadowing). Instead, it is preferable to look to statistical values over a representative area where the BS density $\lambda\left[\mathrm{BS} / \mathrm{km}^{2}\right]$ can be considered uniform.

In this paper, we investigate the experimental distribution of the total electric field due to $2 \mathrm{G}$ to $4 \mathrm{G}$ cellular networks in two Brussels municipalities. By defining a path loss model for propagation, and a Poisson point process for the modeling of macro cell BSs, we show that it is possible to recover this experimental distribution with the SG approach. We then look theoretically at the potential evolution of exposure when the BS network changes according to two scenarios: an increase in macro cell density and the deployment of low-power small cells overlaying current networks.

\section{METHOD}

In this study, we use a model identical to the one we have already used successfully in [10]. The BS pattern is modeled as a homogeneous Poisson Point Process (PPP) $\Phi$ of density $\lambda$, which is a very good approximation when taking into account all BSs of all network providers [11] as must be done 
for exposure assessment. The inter-site distance (ISD) can be computed from

$$
\operatorname{ISD}=\int_{0}^{\infty} 2 \pi \lambda r^{2} \mathrm{e}^{2 \pi \lambda r^{2}} d r=\frac{1}{2 \sqrt{\lambda}} .
$$

For any $\mathrm{BS}$, the power density $S\left[\mathrm{~W} / \mathrm{m}^{2}\right]$ at a horizontal distance $r$ is then given by a simple path loss model

$$
S(r)=\frac{\mathrm{EIRP}}{4 \pi\left(r^{2}+h^{2}\right)^{\alpha / 2}}
$$

where EIRP is the Effective Isotropic Radiated Power, $h$ the height of the BS and $\alpha$ the path loss exponent. Global exposure is characterized by the total power density $S_{W N}$ obtained by summing the power densities from the whole network (WN) of BSs of all network providers, assuming all uncorrelated signals.

$$
S_{W N}=\sum_{i \mid B S_{i} \in \Phi} S\left(r_{i}\right)=\sum_{i \mid B S_{i} \in \Phi} \frac{\mathrm{EIRP}}{4 \pi\left(h^{2}+r_{i}^{2}\right)^{\alpha / 2}} .
$$

Here we assume a simplified homogeneous model, easily applicable to any urban environment, but efficient and leading to reliable results: the BSs are all assumed to be at the same height, isotropic, with the same EIRP. Power density can be translated into root-mean-square electric field strength $[\mathrm{V} / \mathrm{m}]$, which is often more tractable, using

$$
E=\sqrt{Z_{0} S_{W N}}
$$

where $Z_{0}=\sqrt{\mu_{0} / \epsilon_{0}}=120 \pi \approx 377 \Omega$ is the impedance of free-space.

SG provides the theoretical framework to study the statistical properties of (3) over network realizations. The mean of (3), for instance, is given by applying a corollary of Campbell's theorem [12]

$$
\mathbb{E}\left[S_{W N}\right]=\frac{\lambda \mathrm{EIRP}}{2(\alpha-2) h^{\alpha-2}}
$$

while its cumulative distribution function (CDF) is obtained numerically by applying the inversion theorem [13]

$$
F(x)=\frac{1}{2}-\frac{1}{\pi} \int_{0}^{\infty} \frac{\Im\left[\mathrm{e}^{-i t x} \mathcal{L}_{S_{W N}}(-i t)\right]}{t} d t
$$

where $t$ is real and $\mathcal{L}_{S_{W N}}$ is the Laplace transform of (3)

$$
\begin{aligned}
\mathcal{L}_{S_{W N}}(s) & =\mathbb{E}\left[\mathrm{e}^{-s S_{W N}}\right] \\
& =\exp \left[\frac{2 \pi \lambda}{\alpha} \int_{h^{\alpha}}^{\infty}\left(\exp \left(-s \frac{A}{x}\right)-1\right) x^{2 / \alpha-1} \mathrm{~d} x\right] \\
& =\exp \left[\pi \lambda h^{2}\left(1-{ }_{1} F_{1}\left(-2 / \alpha ; 1-2 / \alpha ; \frac{-s A}{h^{\alpha}}\right)\right)\right] \\
& \approx \begin{cases}\exp \left[\pi \lambda h^{2} \sum_{j=1}^{\infty} \frac{2(-s A)^{j}}{h^{\alpha j} j !(j \alpha-2)}\right], & \left|\frac{s A}{h^{\alpha}}\right| \leq c, \\
\exp \left[\pi \lambda h^{2}\left(\frac{-(s A)^{2 / \alpha}}{h^{2}} \Gamma\left(1-\frac{2}{\alpha}\right)+1\right)\right], & \left|\frac{s A}{h^{\alpha}}\right|>c .\end{cases}
\end{aligned}
$$

In this calculation, $A=\mathrm{EIRP} /(4 \pi)$ and ${ }_{1} F_{1}$ is the Kummer confluent hypergeometric function. The last expression corresponds to an approximation for numerical calculations inspired by [14].
When two networks modeled as independent PPPs $\Phi_{1}$ and $\Phi_{2}$ are superimposed, the Laplace transform of the resulting network $\mathcal{L}_{S_{W N}^{t o t}}$ can be written as

$$
\begin{aligned}
\mathcal{L}_{S_{W N}^{\text {tot }}}(s)=\mathbb{E}\left[\mathrm{e}^{\left.-s\left(S_{W N}^{(1)}+S_{W N}^{(2)}\right)\right]}\right. & \mathbb{E}_{\Phi_{1}, A_{1}, \Phi_{2}, A_{2}}\left[\exp \left(-s \sum_{i \in \Phi} \frac{A_{1}}{\left(r_{i}^{2}+h_{1}^{2}\right)^{\alpha_{1} / 2}}\right)\right. \\
& \left.\times \exp \left(-s \sum_{i \in \Phi} \frac{A_{2}}{\left(r_{i}^{2}+h_{2}^{2}\right)^{\alpha_{2} / 2}}\right)\right] \\
= & \mathbb{E}_{\Phi_{1}, A_{1}}\left[\exp \left(-s \sum_{i \in \Phi} \frac{A_{1}}{\left(r_{i}^{2}+h_{1}^{2}\right)^{\alpha_{1} / 2}}\right)\right] \\
& \times \mathbb{E}_{\Phi_{2}, A_{2}}\left[\exp \left(-s \sum_{i \in \Phi} \frac{A_{2}}{\left(r_{i}^{2}+h_{2}^{2}\right)^{\alpha_{2} / 2}}\right)\right] \\
= & \mathcal{L}_{S_{W N}^{(1)}}(s) \times \mathcal{L}_{S_{W N}^{(2)}}(s)
\end{aligned}
$$

where $S_{W N}^{(k)}$ is the total power density due to network $k$ and $\mathcal{L}_{S_{W N}}^{(1)}(s)$ its corresponding Laplace transform. The CDF is then again deduced from (6).

\section{EXPERIMENTAL RESULTS}

In order to fit the parameters in (3), we focused on a zone of the Brussels-Capital Region spreading over two municipalities, Etterbeek and Ixelles. From the knowledge of the exact location of the BSs from all cellular network providers in this zone, the density of macro cell BSs placed on rooftops was deduced: $\lambda=13 \mathrm{BS} / \mathrm{km}^{2}$, which corresponds an ISD of $139 \mathrm{~m}$.

Drive tests were carried out at the beginning of December 2020, during the daytime, in this zone. A spectrum analyzer was placed on the roof of a car riding along the different streets. The entire cellular spectrum was scanned with a resolution of $5 \mathrm{MHz}$ and the three polarization axes were measured and summed up. The power density was obtained for each position of the car, by summing the contribution at each frequency according to

$$
S_{\text {exp }}=\frac{1}{Z_{0}} \sum_{f} E_{f}^{2}
$$

where $E_{f}$ is the electric field strength measured at frequency $f$ belonging to the cellular spectrum detailed in table I. A GPS device gave a position to each measurement, which made it possible to average the measurements over local areas of $2 \mathrm{~m} \times 2 \mathrm{~m}$ to avoid over-representation of some positions. This size was chosen neither too small to smooth out fading, nor too large to keep the spatial sampling relevant. Measurements out of the region under study were removed and statistics of $S_{\text {exp }}$ were derived from the data set. 
TABLE I

Services and frequency bands allocated to all cellular network providers in Brussels.

\begin{tabular}{|l|c|}
\hline Services and frequency bands & Allocated frequencies \\
\hline LTE 800 & $791-821 \mathrm{MHz}$ \\
\hline GSM 900 - UMTS 900 & $925.1-959.9 \mathrm{MHz}$ \\
\hline GSM 1800 - LTE 1800 & $1805-1880 \mathrm{MHz}$ \\
\hline \multirow{2}{*}{ UMTS 2100 } & $2110.3-2140.1 \mathrm{MHz}$ \\
& $2154.9-2169.7 \mathrm{MHz}$ \\
\hline \multirow{2}{*}{ LTE 2600 } & $2620-2640 \mathrm{MHz}$ \\
& $2655-2690 \mathrm{MHz}$ \\
\hline
\end{tabular}

Parameters EIRP, $h$ and $\alpha$ of (3) were then fitted by minimizing

$$
\begin{aligned}
K(\theta) & =\left(\frac{\mu(\theta)}{\mu_{\exp }}-1\right)^{2}+\left(\frac{Q_{10}(\theta)}{Q_{10, \exp }}-1\right)^{2}+\left(\frac{Q_{25}(\theta)}{Q_{25, \exp }}-1\right)^{2} \\
& +\left(\frac{Q_{50}(\theta)}{Q_{50, \exp }}-1\right)^{2}+\left(\frac{Q_{75}(\theta)}{Q_{75, \exp }}-1\right)^{2}+\left(\frac{Q_{90}(\theta)}{Q_{90, \exp }}-1\right)^{2} \\
& +\left(\frac{Q_{95}(\theta)}{Q_{95, \exp }}-1\right)^{2}
\end{aligned}
$$

where $\theta=(h, \alpha, \mathrm{EIRP})$ is the 3 -tuple of parameters. $Q_{x}$ is the $x \%$-quantile and $\mu$ the mean (5) of the distribution of $S_{W N}$ using $\theta$, calculated by (6). The notation " $x_{\text {exp }}$ " refers to statistics obtained from the experimental distribution $S_{\text {exp }}$. The minimization of $K(\theta)$ is an exhaustive search onto a regular grid $\mathcal{G}=\mathcal{I}_{h} \times \mathcal{I}_{\alpha} \times \mathcal{I}_{\text {EIRP }}$ with $\mathcal{I}_{h}=[10 ; 60] \mathrm{m}$ with a step of $1 \mathrm{~m}, \mathcal{I}_{\alpha}=[2 ; 5]$ with a step of 0.02 and $\mathcal{I}_{\text {EIRP }}=[60.0 ; 85.0] \mathrm{dBm}$ with a step of $0.05 \mathrm{dBm}$.

Statistical parameters of the SG and experimental distributions are listed in table II. The optimal set of parameters for the propagation model (3) is also shown. As seen, fitted parameters are realistic on physical ground. Fig. 1 clearly shows that the experimental and the SG CDFs are very close, which is also evidenced by the Kolmogorov-Smirnov (KS) distance of 0.02 . It can be concluded that SG in combination with (3) provides a realistic statistical model of the total exposure.

\section{IMPACT OF BS DENSITY}

Using parameter values of Table II, the impact of an increase of the number of BSs in the network can be theoretically studied. First, we considered that macro cells $(m c)$ identical to those being already part of the network are introduced. We increased the density of macro cells of the PPP from $\lambda_{m c, 0}=13 \mathrm{BS} / \mathrm{km}^{2}$ to $\lambda_{m c}=25 \mathrm{BS} / \mathrm{km}^{2}$ and $50 \mathrm{BS} / \mathrm{km}^{2}$. In this scenario, two extreme cases were considered: keeping the EIRP constant and having an EIRP evolving as

$$
\operatorname{EIRP}_{m c}=\frac{\operatorname{EIRP}_{m c, 0}}{\left(\lambda_{m c} / \lambda_{m c, 0}\right)^{\alpha / 2}},
$$

where $\operatorname{EIRP}_{m c, 0}=83.65 \mathrm{dBm}$, to ensure same power density at cell edge for all $\lambda_{m c}$. Secondly, we considered that a new network of small cells $(s c)$, independent of the network of
TABLE II

PARAMETERS OF THE STATISTICAL DISTRIBUTIONS OF THE POWER DENSITY FOR THE WHOLE CELLULAR SPECTRUM, IN THE BRUSSELS-CAPITAL REgION (IXELLES AND ETTERBEEK). EXP: EXPERIMENTAL RESULTS. SG: CDF OBTAINED BY A NUMERIC GIL-PELAEZ INVERSION. $Q_{x}$ 'S ARE THE QUANTILES, $\mu$ THE MEAN.

\begin{tabular}{|l||c|c|}
\hline \multicolumn{1}{|c||}{} & Exp & SG \\
\hline$h(\mathrm{~m})$ & \multicolumn{2}{|c|}{54} \\
$\alpha$ & \multicolumn{2}{|c|}{3.62} \\
EIRP $(\mathrm{dBm})$ & \multicolumn{2}{c|}{83.65} \\
\hline$Q_{05}\left(\mathrm{~W} / \mathrm{m}^{2}\right)$ & $1.02 \cdot 10^{-4}$ & $1.12 \cdot 10^{-4}$ \\
$Q_{10}\left(\mathrm{~W} / \mathrm{m}^{2}\right)$ & $1.31 \cdot 10^{-4}$ & $1.48 \cdot 10^{-4}$ \\
$Q_{25}\left(\mathrm{~W} / \mathrm{m}^{2}\right)$ & $2.53 \cdot 10^{-4}$ & $2.59 \cdot 10^{-4}$ \\
$Q_{50}\left(\mathrm{~W} / \mathrm{m}^{2}\right)$ & $6.33 \cdot 10^{-4}$ & $5.72 \cdot 10^{-4}$ \\
$Q_{75}\left(\mathrm{~W} / \mathrm{m}^{2}\right)$ & $1.66 \cdot 10^{-3}$ & $1.55 \cdot 10^{-3}$ \\
$Q_{90}\left(\mathrm{~W} / \mathrm{m}^{2}\right)$ & $3.79 \cdot 10^{-3}$ & $3.97 \cdot 10^{-3}$ \\
$Q_{95}\left(\mathrm{~W} / \mathrm{m}^{2}\right)$ & $5.90 \cdot 10^{-3}$ & $6.32 \cdot 10^{-2}$ \\
$\mu\left(\mathrm{W} / \mathrm{m}^{2}\right)$ & $1.51 \cdot 10^{-3}$ & $1.40 \cdot 10^{-3}$ \\
KS distance & \multicolumn{2}{|c}{0.03} \\
\hline \multicolumn{2}{|c}{} \\
\hline \multicolumn{2}{|c}{}
\end{tabular}

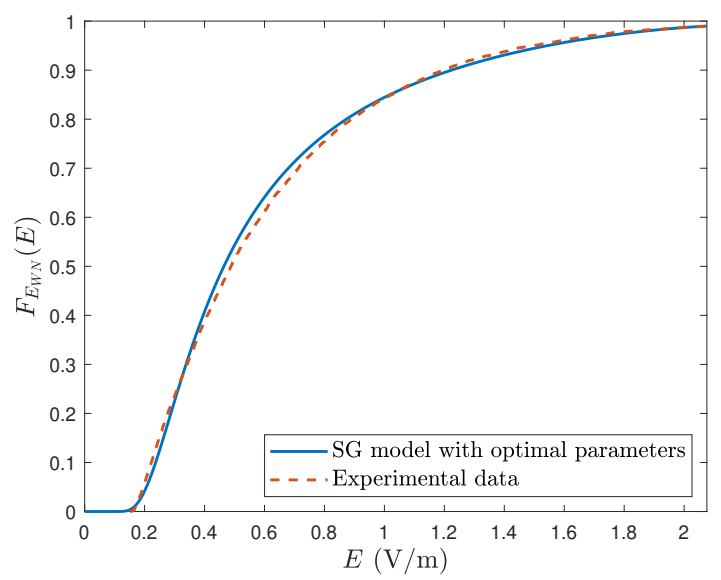

Fig. 1. CDF of $E=\sqrt{Z_{0} S_{\text {exp }}}$ for the network made of BSs from all network providers in Brussels for the whole cellular spectrum.

macro cells, is being introduced. We assumed that the small cell network is also modeled by (3). Under these hypotheses, the Laplace transform of the total power density due to the resulting network can be calculated by (8) and the CDF can be obtained by (6). The expected value of the total power density is given by the sum of the expected values of the power density of both networks. Small cells are considered as lowpower transmitters placed on street furniture and belonging to category E2 [15], what does not require a permit to be placed especially in Brussels, Belgium and in France [16]. We therefore set $h_{s c}=3 \mathrm{~m}, \alpha_{s c}=2.1$ and $\mathrm{EIRP}_{s c}=33 \mathrm{dBm}$. The mean, the median and the probabilities to reach $1 \mathrm{~V} / \mathrm{m}$, $3 \mathrm{~V} / \mathrm{m}$ and $6 \mathrm{~V} / \mathrm{m}$ thresholds are listed in Table III, Table IV and Table $\mathrm{V}$ for the case of a densification of macro cell network, keeping the EIRP constant and not, and then for the case of the addition of a network of small cells, respectively. The CDFs are shown in Fig. 2, Fig. 3 and Fig. 4, respectively.

As can be clearly seen, assuming new macro cell deployments with the same EIRP, exposure increases significantly, while assuming a decrease in EIRP, global exposure decreases. 


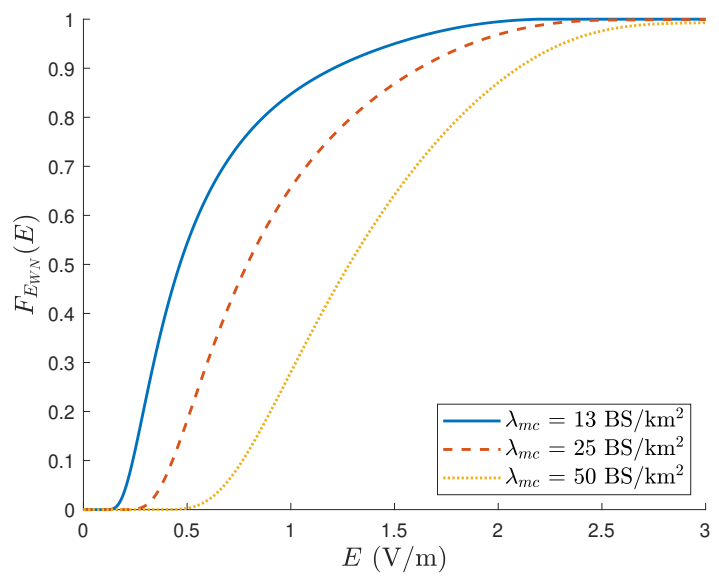

Fig. 2. Impact of the densification of the network of macro cells, keeping the EIRP constant.

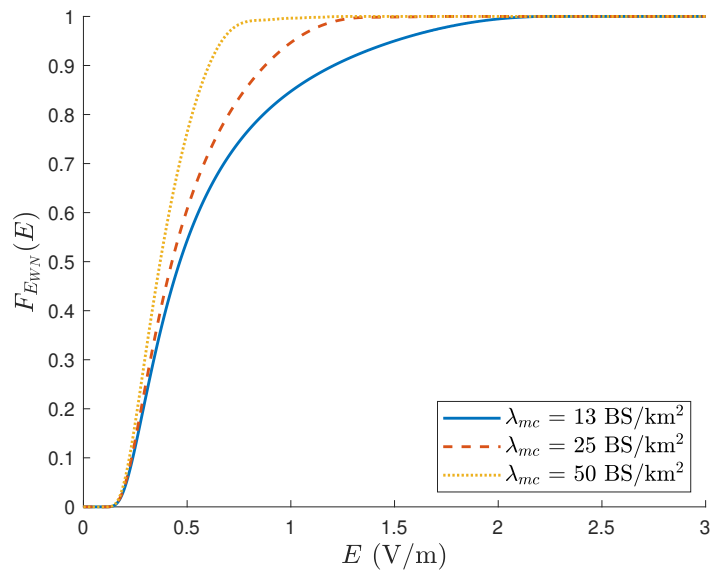

Fig. 3. Impact of the densification of the network of macro cells, reducing the EIRP.

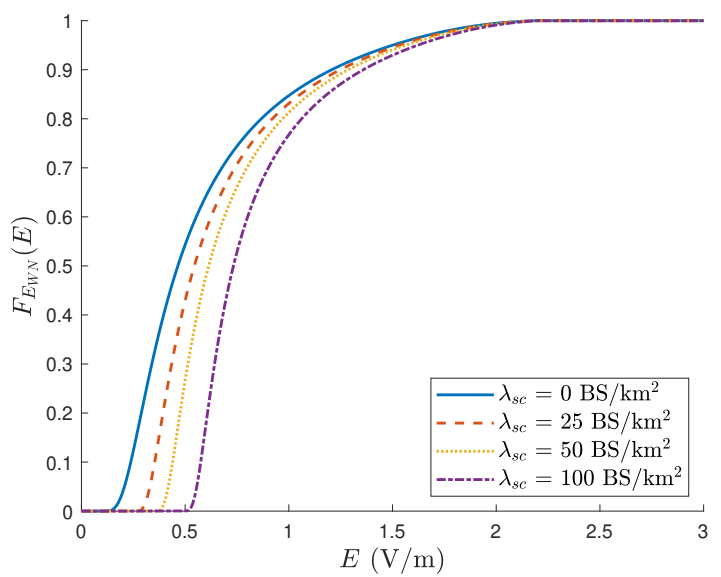

Fig. 4. Impact of the densification of the addition of a new network of small cells.
TABLE III

EVOLUTION OF THE MEDIAN OF THE DISTRIBUTION AND PROBABILITIES TO REACH EXPOSURE THRESHOLDS WHEN THE MACRO CELL DENSITY INCREASES, KEEPING THE EIRP CONSTANT.

\begin{tabular}{|l||c|c|c|}
\hline$\lambda_{m c}\left(\mathrm{BS} / \mathrm{km}^{2}\right)$ & 13 & 25 & 50 \\
\hline$Q_{50}(\mathrm{~V} / \mathrm{m})$ & 0.46 & 0.79 & 1.28 \\
$\mu\left(\mathrm{mW} / \mathrm{m}^{2}\right)$ & 1.40 & 2.79 & 5.58 \\
$\mathbf{P}[E>1 \mathrm{~V} / \mathrm{m}](\%)$ & 15.27 & 34.34 & 71.98 \\
$\mathbf{P}[E>3 \mathrm{~V} / \mathrm{m}](\%)$ & $<10^{-2}$ & $<10^{-2}$ & 0.05 \\
$\mathbf{P}[E>6 \mathrm{~V} / \mathrm{m}](\%)$ & $<10^{-2}$ & $<10^{-2}$ & $<10^{-2}$ \\
\hline
\end{tabular}

TABLE IV

EVOLUTION OF THE MEDIAN OF THE DISTRIBUTION AND PROBABILITIES TO REACH EXPOSURE THRESHOLDS WHEN THE MACRO CELL DENSITY INCREASES, KEEPING THE EIRP CONSTANT.

\begin{tabular}{|l||c|c|c|}
\hline$\lambda_{m c}\left(\mathrm{BS} / \mathrm{km}^{2}\right)$ & 13 & 25 & 50 \\
\hline$Q_{50}(\mathrm{~V} / \mathrm{m})$ & 0.46 & 0.43 & 0.37 \\
$\mu\left(\mathrm{mW} / \mathrm{m}^{2}\right)$ & 1.40 & 0.82 & 0.47 \\
$\mathbf{P}[E>1 \mathrm{~V} / \mathrm{m}](\%)$ & 15.27 & 5.33 & 0.35 \\
$\mathbf{P}[E>3 \mathrm{~V} / \mathrm{m}](\%)$ & $<10^{-2}$ & $<10^{-2}$ & $<10^{-2}$ \\
$\mathbf{P}[E>6 \mathrm{~V} / \mathrm{m}](\%)$ & $<10^{-2}$ & $<10^{-2}$ & $<10^{-2}$ \\
\hline
\end{tabular}

TABLE V

EVOLUTION OF THE MEDIAN OF THE DISTRIBUTION AND PROBABILITIES TO REACH EXPOSURE THRESHOLDS WHEN A SMALL CELL NETWORK IS SUPERIMPOSED ON THE CURRENT NETWORK.

\begin{tabular}{|l||c|c|c|c|}
\hline$\lambda_{s c}\left(\mathrm{BS} / \mathrm{km}^{2}\right)$ & 0 & 25 & 50 & 100 \\
\hline$Q_{50}(\mathrm{~V} / \mathrm{m})$ & 0.46 & 0.54 & 0.61 & 0.73 \\
$\mu\left(\mathrm{mW} / \mathrm{m}^{2}\right)$ & 1.40 & 1.64 & 1.86 & 2.31 \\
$\mathbf{P}[E>1 \mathrm{~V} / \mathrm{m}](\%)$ & 15.27 & 16.88 & 18.71 & 23.24 \\
$\mathbf{P}[E>3 \mathrm{~V} / \mathrm{m}](\%)$ & $<10^{-2}$ & $<10^{-2}$ & $<10^{-2}$ & $<10^{-2}$ \\
$\mathbf{P}[E>6 \mathrm{~V} / \mathrm{m}](\%)$ & $<10^{-2}$ & $<10^{-2}$ & $<10^{-2}$ & $<10^{-2}$ \\
\hline
\end{tabular}

Interestingly, the addition of a network of small cells causes a slight change in the head of the distribution but leaves its tail unchanged. This can also be seen in Fig. 5 where the mean total power density (5) is shown as a function of the macro cell density for the first scenario and as a function of the small cell density for the second scenario. The most realistic evolution of the network to meet the growing user connection demand is a combination of increasing the density of macro cells, with a decreasing EIRP, and adding a new network of small cells to support it. This simplified study where all small and macro cells are identical and where beamforming is not taken into account, leads to believe that global exposure due to cellular networks does not change significantly in such a scenario.

\section{CONCLUSION}

Exposure to electromagnetic fields was modeled simply and reliably for the entire cellular spectrum by using SG with a simple path loss model. The approach followed was based on the Gil-Pelaez inversion of the Laplace transform of the total network power density. The impact of BS densification was studied according to three cases: the addition of BSs identical to those of the current network, with constant EIRP or density-varying EIRP, and the addition of a new network of small cells. In particular, it can be concluded that only the macro cell network has an effect on the tail of the exposure distribution in an urban environment. Global 


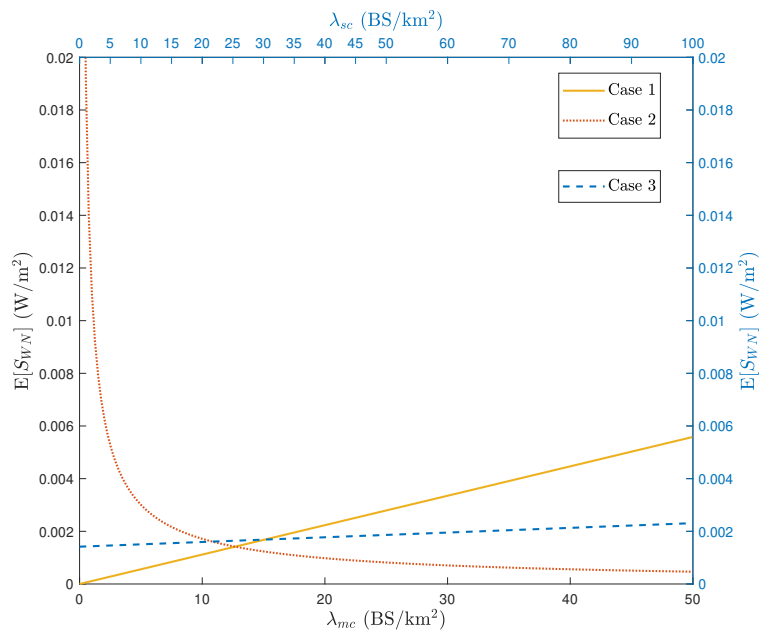

Fig. 5. Mean total power density (5) as a function of the cell density. Case 1: Densification of macro cells at constant EIRP. Case 2: Densification of macro cells with a varying EIRP. Case 3: Densification of small cells with a macro cell density $\lambda_{m c}=13 \mathrm{BS} / \mathrm{km}^{2}$. Cases 1 and 2 vary along the lower axis and case 3 along the upper axis. The current mean power density used in Table II corresponds to the intersection between curves 1 and 2 .

exposure remains nearly unchanged if the network evolution implies a smart densification of macro cells in combination to the deployment of category E2 micro-cells at the street level.

\section{REFERENCES}

[1] D. Moltchanov, "Distance distributions in random networks," Ad Hoc Networks, vol. 10, no. 6, pp. 1146-1166, 2012.

[2] H. ElSawy, A. Sultan-Salem, M. Alouini, and M. Z. Win, "Modeling and analysis of cellular networks using stochastic geometry: A tutorial," IEEE Communications Surveys Tutorials, vol. 19, no. 1, pp. 167-203, 2017.

[3] R. Mathar and J. Mattfeldt, "On the distribution of cumulated interference power in rayleigh fading channels.," Wireless Networks, vol. 1, no. 1, pp. 31-36, 1995

[4] C. E. O'Lone, H. S. Dhillon, and R. M. Buehrer, "A statistical characterization of localization performance in wireless networks," IEEE Transactions on Wireless Communications, vol. 17, no. 9, pp. 58415856, 2018.

[5] I. Nakata and N. Miyoshi, "Spatial stochastic models for analysis of heterogeneous cellular networks with repulsively deployed base stations," Performance Evaluation, vol. 78, pp. 7-17, 2014.

[6] M. Filo, C. H. Foh, S. Vahid, and R. Tafazolli, "Stochastic geometry analysis of ultra-dense networks: Impact of antenna height and performance limits," ArXiv, vol. abs/1712.02235, 2017.

[7] Y. Zeng, R. Zhang, and T. J. Lim, "Wireless communications with unmanned aerial vehicles: opportunities and challenges," IEEE Communications Magazine, vol. 54, p. 36-42, May 2016.

[8] J. a. Valente, D. Sanz, A. Barrientos, J. d. Cerro, A. Ribeiro, and C. Rossi, "An air-ground wireless sensor network for crop monitoring," Sensors, vol. 11, p. 6088-6108, Jun 2011.

[9] M. A. Hajj, S. Wang, P. De Doncker, C. Oestges, and J. Wiart, "A Statistical Estimation of 5G Massive MIMO's Exposure using Stochastic Geometry," in 2020 XXXIIIrd General Assembly and Scientific Symposium of the International Union of Radio Science, pp. 1-3, 2020.

[10] Q. Gontier, F. Rottenberg, L. Petrillo, F. Horlin, J. Wiart, C. Oestges, and P. De Doncker, "A stochastic geometry approach to EMF exposure modeling," 2021. Submitted.
[11] J. S. Gomez, A. Vasseur, A. Vergne, P. Martins, L. Decreusefond, and W. Chen, "A Case Study on Regularity in Cellular Network Deployment," IEEE Wireless Communications Letters, vol. 4, pp. 421424, Aug 2015.

[12] J. F. C. Kingman, Poisson processes, vol. 3 of Oxford Studies in Probability, ch. Sums over Poisson processes, pp. 28-29. New York: The Clarendon Press Oxford University Press, 1993. Oxford Science Publications.

[13] J. Gil-Pelaez, "Note on the inversion theorem," Biometrika, vol. 38, pp. 481-482, 121951.

[14] A. Aravanis, T. Tu, O. Muñoz, A. Pascual-Iserte, and M. Di Renzo, "A tractable closed form approximation of the ergodic rate in Poisson cellular networks," EURASIP Journal on Wireless Communications and Networking, vol. 12, 122019.

[15] ITU, " $5 \mathrm{G}$ technology and human exposure to radio frequency electromagnetic fields," 2019. 05/2019, Supplement 9.

[16] GSMA, "Improving wireless connectivity through small cell deployment," December 2016 